\title{
Novel Endoscopic Technique for the Diagnosis of Early Gastric Cancer: Endoscopy with Crystal Violet Staining
}

\author{
Ken Ohata ${ }^{a}$ Ryoichi Misaka ${ }^{b}$ Takafumi Ito $^{a}$ Hideyuki Chiba ${ }^{a}$ Yosuke Tsuji ${ }^{a}$ \\ Makoto Yamawaki $^{a}$ Tomohiko Ohya ${ }^{c}$ Masahiko Inamori ${ }^{d}$ Atsushi Nakajima ${ }^{d}$ \\ Nobuyuki Matsuhashi ${ }^{\mathrm{a}}$ \\ ${ }^{a}$ Department of Gastroenterology, Kanto Medical Center, NTT East, ${ }^{b}$ Department of Gastroenterology, Institute of \\ Geriatrics, Tokyo Women's Medical University, and 'Department of Endoscopy, The Jikei University School of \\ Medicine, Tokyo, and d Gastroenterology Division, Yokohama City University School of Medicine, Yokohama, Japan
}

Dear Sir,

Recently, endoscopic submucosal dissection (ESD) has been established as a minimally invasive technique for en bloc removal of gastric cancer [1]. Conventional endoscopy and chromoendoscopy with indigo carmine dye are usually performed for preoperative assessment of the lateral extent of early gastric cancer before ESD [2]. In some cases, however, accurate diagnosis of early gastric cancer remains difficult, even for an expert endoscopist. Even when an en block resection is completed, inaccurate identification of the margin line or inaccurate estimation of tumor depth may lead to lateral or horizontal margin positivity, and/or ESD failure.

A 77-year-old man was hospitalized for endoscopic resection of an early gastric cancer. The tumor was located in the lesser curvature of the gastric corpus. Conventional white-light endoscopy and chromoendoscopy with indigo carmine dye revealed a slightly elevated and reddened area with a biopsy ulcer. The lateral margin, however, could not be visualized clearly (fig. 1a, b). After washing off indigo carmine dye, we sprayed $0.03 \%$ crystal violet solution on and around the tumor. Af- ter crystal violet staining, the entire cancer region showed faint staining, while the surrounding noncancerous mucosa was densely stained (fig. 1c). Thus, we could clearly recognize the actual border line between the normal mucosa and the cancer, and the resection line could be determined. In this way, we could perform successful ESD (fig. 1d, e). The resected specimen showed well-differentiated adenocarcinoma confined to the mucosal layer with a sufficient cancer-free distance $(>5 \mathrm{~mm})$ from the sample edge (fig. 1f).

To the best of our knowledge, the technique of chromoendoscopy with crystal violet staining for visualization of the outline of gastric cancer has not yet been reported. The difference in the intensity of crystal violet staining between cancerous tissue and intestinal metaplasia or background mucosa is caused by the difference in the degree of dye absorption. Other diagnostic techniques for gastric neoplasms utilizing a narrow band imaging system and magnifying scope have also been described [3], but they are not always useful, and these devices are somewhat cumbersome to use.
Our novel technique is simple, easy to perform and allows good visualization in many cases. We propose this technique as an easy and effective tool in determining the precise lateral margin of early gastric cancer.

\section{References}

1 Oka S, Tanaka S, Kaneko I, Mouri R, Hirata M, Kawamura T, Yoshihara M, Chayama K: Advantage of endoscopic submucosal dissection compared with EMR for early gastric cancer. Gastrointest Endosc 2006;64:877883.

2 Yamashita H, Kitayama J, Nagawa H: Chromoendoscopy with indigo carmine dye added to acetic acid in the diagnosis of gastric neoplasia. Gastrointest Endosc 2009;69: 1407-1408.

3 Yao K, Anagnostopoulos GK, Ragunath K: Magnifying endoscopy for diagnosing and delineating early gastric cancer. Endoscopy 2009;41:462-467.

\section{KARGER}

Fax +41613061234 E-Mail karger@karger.ch www.karger.com
M. Inamori, $\mathrm{MD}, \mathrm{PhD}$

Gastroenterology Division, Yokohama City University Hospital

3-9 Fukuura, Kanazawa-ku, Yokohama 236-0004 (Japan)

Tel. +81 45787 2640, Fax +81 457843546

E-Mailinamorim@med.yokohama-cu.ac.jp 


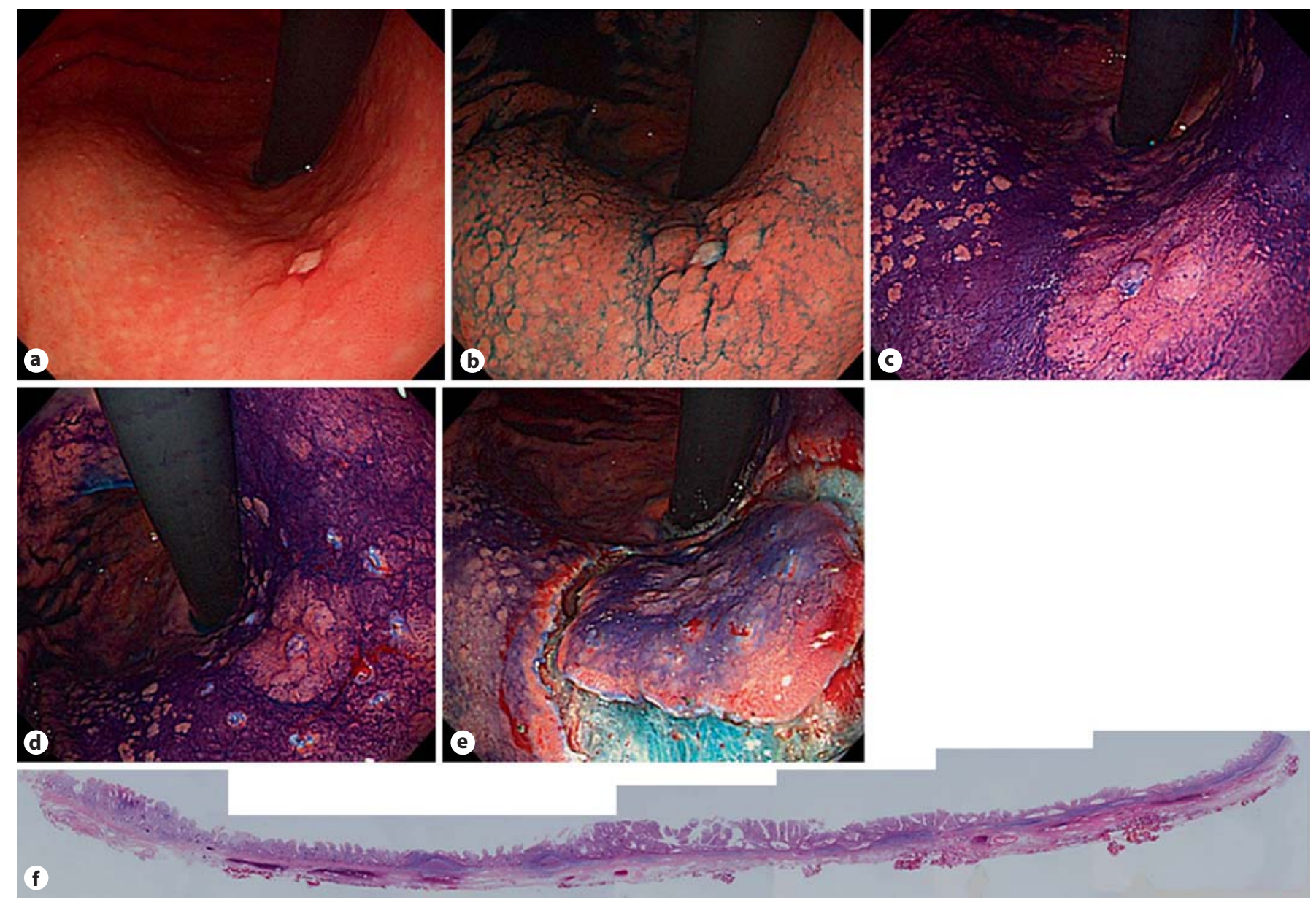

Fig. 1. a Endoscopic view of a gastric cancer located in the lesser curvature of the gastric corpus. $\mathbf{b}$ Chromoendoscopy with indigo carmine dye; it was difficult to recognize the tumor-negative lateral margin clearly. c After crystal violet staining, the border of the lesion was easily visualized. d, e Endoscopic submucosal dis-

section performed after crystal violet staining. $f$ The resected specimen showed well-differentiated adenocarcinoma confined to the mucosal layer with a sufficient cancer-free distance ( $>5 \mathrm{~mm})$ from the sample edge. 